\title{
Beneficial Effects of Incretin-Based Therapy on Glycemic Control, Adipokines, Insulin Sensitivity Parameters and Weight Loss in Overweight or Obese Patients With Newly Diagnosed Type 2 Diabetes: A Prospective Study
}

\author{
Yang Yu ${ }^{\mathrm{a}}$, Xiaohui Yang ${ }^{\mathrm{a}, \mathrm{b}}$, Xiaomei Wang ${ }^{\mathrm{a}}$, Tao Zhang ${ }^{\mathrm{a}}$, Miao Sui ${ }^{\mathrm{a}}$, \\ Jingyao Liu ${ }^{\mathrm{a}}$, Jingyi $\mathrm{Xu}^{\mathrm{a}}$, Yu Hou ${ }^{\mathrm{a}}$
}

\begin{abstract}
Background: The aim of the study was to examine the incretin-based therapy on glycemic control, insulin sensitivity parameters, weight loss and adipokines in overweight or obese patients with newly diagnosed type 2 diabetes mellitus (T2DM).

Methods: This was a 24-week prospective study of 75 enrolled overweight or obese subjects (body mass index (BMI) $\geq 25 \mathrm{~kg} / \mathrm{m}^{2}$ ) with newly diagnosed T2DM randomly assigned to the liraglutide, metformin, and sitagliptin groups. Outcome measures were fasting blood glucose (FBG), HbA1c, weight, BMI, waist circumstance, plasma lipids, fast insulin, fast C-peptide, HOMA-IR, HOMA-B, leptin, adiponectin, and high-sensitivity $\mathrm{C}$-reactive protein (hsCRP).

Results: At the endpoint of 24 weeks, administration of liraglutide, metformin or sitagliptin all resulted in significant improvements of glycemic control, weight loss and insulin sensitivity parameters. It was also demonstrated that liraglutide was superior to metformin and sitagliptin in terms of achieving target $\mathrm{HbAlc}$ values and sustaining weight loss after 24-week treatment, but not to the incidence of adverse events (AEs). Moreover, liraglutide administration showed beneficial effects on reducing leptin levels and L/A ratio as well as elevating adiponectin levels compared to the metformin and sitagliptin administration.
\end{abstract}

Conclusion: Liraglutide treatment during 24 weeks in newly diagnosed T2DM patients led to reduction of BMI and improvement of glucose control, insulin sensitivity and resistance parameters. Additionally, circulating levels of adipokines could play an important role in GLP-1 treatment.

Keywords: Incretin; T2DM; Insulin sensitivity; Adipokine

Manuscript accepted for publication August 21, 2015

aEndocrinology Department of Affiliated Zhongshan Hospital of Dalian University, DaLian, Liaoning 116001, China

${ }^{b}$ Corresponding Author: Xiaohui Yang, Endocrinology Department of Affiliated Zhongshan Hospital of Dalian University, DaLian, Liaoning 116001, China. Email: xhyang8899@163.com

doi: http://dx.doi.org/10.14740/jem304w
Introduction

Type 2 diabetes mellitus (T2DM) is a complex metabolic condition, resulting in a hyperglycemia status. The insulin resistance, a subnormal response to insulin-mediated cellular actions, along with relative pancreatic $\beta$-cell insufficiency, is the essential features in the pathogenesis of T2DM [1].

Overweight and obesity are widely believed to contribute to the development of several metabolic complications, including insulin resistance and T2DM [1, 2]. However, the pathophysiological link between obesity and insulin resistance remains to be elucidated, but several recent studies indicate a central role for adipose tissue and its endocrine dysfunction. Adipose tissue is now considered as an active secretary organ, sending out and responding to signals that modulate appetite, insulin sensitivity, energy expenditure, inflammation and immunity. Obesity and insulin resistance are often associated with a low-grade inflammatory state in adipose tissue. Adipose tissue produces several proteins (adipokines) such as leptin and adiponectin that modulate insulin sensitivity and appear to play an important role in the pathogenesis of insulin resistance [3-5].

Metformin is recommended as the first-line oral antihyperglycemic agent for T2DM in the American Diabetes Association (ADA)/European Association for the Study of Diabetes (EASD) guidelines, when lifestyle fails to keep HbAlc at target, especially in the overweight and obese subjects [6]. The endogenous incretin GLP-1, a kind of peptide hormone secreted from endocrine cells in the small intestine, is demonstrated to activate insulin secretion, inhibit glucagon secretion, slow gastric emptying, and induce weight loss. Recently, incretin-based agents, GLP-1 receptor agonists (GLP-1RA) and dipeptidyl peptidase-4 inhibitors (DPP4i), a logical choice of restoring $\beta$-cell function and decreasing insulin resistance, have been developed to treat T2DM [7]. Many studies indicated that both GLP-1RA and DPP4i have the priority over metformin and sulfonylurea in reducing plasma glucose and body weight, and increasing insulin sensitivity. However, these studies seldom select the newly diagnosed diabetic patients as subjects, and rarely evaluate the adipokines profile [8-11]. To our knowledge, no studies have addressed the effect of liraglutide and sitagliptin treatment on plasma glycemic control, weight loss and circulating adipokines in newly diagnosed diabetic patients in daily clinical practice.

We reported the results of a 24 -week, open-label, randomized, prospective study designed to investigate the relation- 
ship of glycemic control, weight, insulin resistance indexes (HOMA-IR and HOMA-B) and serum adipokines (leptin and adiponectin) in newly diagnosed overweight or obese T2DM patients at the beginning and 24 weeks after the initiation of liraglutide, sitagliptin or metformin in daily clinical practice.

\section{Methods}

\section{Participants}

Participants aged 18 - 65 years with T2DM diagnosed within 3 months were eligible if their HbA1c value was $7.0-11.0 \%$ and if they had a BMI of $25.0-35.0 \mathrm{~kg} / \mathrm{m}^{2}$. All subjects were naive to any hypoglycemic treatment.

Exclusion criteria included previous hypoglycemic treatment, liver or renal function, clinically significant cardiovascular disease, retinopathy or maculopathy requiring acute treatment, uncontrolled hypertension $(\geq 180 / 110 \mathrm{~mm} \mathrm{Hg})$, or cancer. Further exclusion criteria included current use of any systemic or topical treatment with drugs known to influence glucose metabolism or weight (systemic glucocorticoids, nonselective $\beta$-sympathetic blockers, and weight loss drugs within 3 months of randomization), anemia, a personal or family history of medullary thyroid carcinoma or multiple endocrine neoplasia syndrome type 2, or a history of drug or alcohol abuse.

All participants provided written consent before any procedures. The trial design was approved by the local ethics committee review board and was conducted according to Good Clinical Practice in accordance with the Declaration of Helsinki.

\section{Trial design}

A 24-week, randomized, open-label, parallel clinical trial was conducted at the Endocrinology Department of Zhongshan Hospital affiliated to Dalian University. Recruitment occurred from March 2013 to November 2014. Participants were randomly assigned to subcutaneous liraglutide, or oral sitagliptin or metformin on the basis of computer-generated random numbers by a person not involved in the recruitment. Liraglutide was administered subcutaneously at a dose of $0.6 \mathrm{mg}$ in the morning for 1 week followed by an increase to $1.2 \mathrm{mg}$ once daily for the next 23 weeks or $1.8 \mathrm{mg}$ if necessary. Sitagliptin was taken $100 \mathrm{mg}$ once daily in the morning, and metformin dosage ranged from 1,500 to $2,000 \mathrm{mg}$ daily.

The glycemic control target was defined as HbAlc $<7 \%$ and fasting blood glucose $(\mathrm{FBG}) \leq 7.0 \mathrm{mmol} / \mathrm{L}$. Postprandial blood glucose was not chosen for variable factors. During the 24-week treatment period, subjects were required to visit the outpatient once every 2 weeks, and telephone contact every week. Lifestyle intervention was not actively promoted.

\section{Assessments}

At the baseline and the endpoint of 24 weeks, blood pressure, waist circumference, height and weight were recorded for each subject, and BMI was calculated as $\mathrm{kg} / \mathrm{m}^{2}$. Clinical parameters evaluated at baseline and the endpoint of 24 weeks included HbA1c, fatty acids, FBG, fast insulin and fast C-peptide. The enrolled subjects were provided with a commercial glucose meter (Accu-Chek, Roche Diagnostics, Mannheim, Germany) and a diary to record their seven-point (before and $2 \mathrm{~h}$ after breakfast, lunch, dinner and $10 \mathrm{pm}$ of bedtime) profile of selfmonitored blood glucose (SMBG) once per week.

Hypoglycemic episodes and adverse events (AEs) were recorded throughout the study. Hypoglycemia was determined by plasma glucose $\leq 3.9 \mathrm{mmol} / \mathrm{L}$, or occurrences of definite hypoglycemic symptoms. Hypoglycemia was considered severe when the event required third party assistance. AEs were classified as serious if they resulted in death, life-threatening experiences, hospitalization, or persistent of significant disability or incapacity.

Plasma was separated from blood within $30 \mathrm{~min}$ and stored at $-70{ }^{\circ} \mathrm{C}$ until analysis. Plasma glucose was determined by the glucose oxidase technique. Plasma total cholesterol, highdensity lipoprotein (HDL) cholesterol, and triglycerides (TG) were measured with an autoanalyzer (Hitachi 7150; Hitachi, Tokyo, Japan) using an enzymatic colorimetric method. Lowdensity lipoprotein (LDL) cholesterol was calculated using the Friedewald formula, and high-sensitivity C-reactive protein (hsCRP) was determined with a BN II analyzer (DadeBehring, Newarkand, DE, USA) using a latex-enhanced immunonephelometric method. Serum insulin and C-peptide concentrations were measured using Immulite 2000 solid-phase chemiluminescent immunometric assays (Immulite 2000; Siemens, Erlangen, Germany). Blood pressure was measured using semiautomatic upper arm blood pressure monitor (Boso Mercurius E; Bosch+Sohn, Jungingen, Germany). Plasma adiponectin (B-Bridge International, Sunnyvale, CA, USA) and leptin (R\&D Systems, Minneapolis, MN, USA) were measured using enzyme-linked immunosorbent assays.

\section{Statistical analysis}

All data were presented as the mean and standard deviation or $\mathrm{n}$ and \%, and were analyzed using SPSS windows version 19.0. The distribution of variables was analyzed with KolmogorovSmirnov test. Changes in parameters from the baseline values within group were evaluated using two-tailed paired $t$-test. One-way univariate analysis of variance (ANOVA) was used to compare the differences in clinical characteristics among the three groups at baseline and after treatment assessed for significance using for the discrete or continuous data and the Chi-square test for frequency distributions. P-value $<0.05$ was considered to be statistically significant.

\section{Results}

\section{Subjects}

A total of 75 T2DM patients $(49.3 \%$ females $)$ with a mean 
Table 1. Baseline Demographics and Characteristics of the Study Participants

\begin{tabular}{|c|c|c|c|c|}
\hline & Liraglutide group & Metformin group & Sitagliptin group & P value \\
\hline $\mathrm{N}$ & 24 & 26 & 25 & NS \\
\hline $\operatorname{Sex}(M / F)$ & $12 / 12$ & $13 / 13$ & $13 / 12$ & NS \\
\hline Age (years) & $56.4 \pm 8.6$ & $56.7 \pm 9.1$ & $55.3 \pm 9.5$ & NS \\
\hline Duration of diabetes (years) & $6.4 \pm 2.1$ & $6.2 \pm 2.5$ & $6.5 \pm 2.2$ & NS \\
\hline Waist circumstance (cm) & $110.2 \pm 4.7$ & $109.7 \pm 4.2$ & $110.1 \pm 3.8$ & NS \\
\hline WHR & $0.89 \pm 0.08$ & $0.89 \pm 0.06$ & $0.89 \pm 0.06$ & NS \\
\hline BMI $\left(\mathrm{kg} / \mathrm{m}^{2}\right)$ & $30.4 \pm 2.7$ & $30.2 \pm 2.4$ & $30.6 \pm 3.2$ & NS \\
\hline Weight (kg) & $88.7 \pm 6.5$ & $88.2 \pm 7.6$ & $88.9 \pm 6.2$ & NS \\
\hline HbA1c (\%) & $8.8 \pm 1.0$ & $8.7 \pm 0.7$ & $8.6 \pm 0.8$ & NS \\
\hline FBG $(\mathrm{mmol} / \mathrm{L})$ & $9.3 \pm 1.6$ & $9.3 \pm 0.9$ & $9.2 \pm 1.7$ & NS \\
\hline Fast insulin $(\mu \mathrm{IU} / \mathrm{mL})$ & $13.7 \pm 4.4$ & $12.8 \pm 4.9$ & $13.9 \pm 4.4$ & NS \\
\hline Fast C peptide (ng/mL) & $3.0 \pm 1.2$ & $3.1 \pm 1.0$ & $3.0 \pm 1.1$ & NS \\
\hline HOMA-IR (mol mIU/L²) & $5.8 \pm 2.4$ & $5.3 \pm 2.1$ & $5.8 \pm 2.4$ & NS \\
\hline HOMA-B ( $\mu \mathrm{IU} / \mathrm{mmol})$ & $49.3 \pm 16.6$ & $45.2 \pm 18.4$ & $51.3 \pm 18.7$ & NS \\
\hline Cholesterol (mmol/L) & $5.7 \pm 0.8$ & $5.8 \pm 1.0$ & $5.9 \pm 0.8$ & NS \\
\hline Triglycerides (mmol/L) & $2.7 \pm 1.1$ & $2.9 \pm 0.9$ & $2.6 \pm 1.1$ & NS \\
\hline HDL-c (mmol/L) & $1.4 \pm 0.5$ & $1.5 \pm 0.5$ & $1.5 \pm 0.4$ & NS \\
\hline LDL-c (mmol/L) & $3.4 \pm 0.6$ & $3.6 \pm 0.7$ & $3.4 \pm 0.7$ & NS \\
\hline Leptin (ng/dL) & $28.4 \pm 9.2$ & $29.7 \pm 5.6$ & $27.6 \pm 7.9$ & NS \\
\hline Adiponectin $(\mu \mathrm{g} / \mathrm{dL})$ & $6.5 \pm 2.2$ & $6.3 \pm 1.6$ & $6.8 \pm 1.8$ & NS \\
\hline $\mathrm{L} / \mathrm{A}$ ratio $(\mathrm{ng} / \mu \mathrm{g})$ & $5.1 \pm 3.2$ & $5.1 \pm 1.8$ & $4.5 \pm 2.1$ & NS \\
\hline hsCRP (mg/L) & $2.6 \pm 1.8$ & $2.6 \pm 1.2$ & $2.4 \pm 1.7$ & NS \\
\hline
\end{tabular}

Values are represented as mean \pm standard variation. NS: non-significance.

age of $56.1 \pm 9.0$ years and mean $\mathrm{HbA} 1 \mathrm{c}$ of $8.7 \pm 0.8 \%$ were enrolled. One male patient was excluded from the liraglutide group due to severe gastrointestinal side effects, and another female patient was lost due to protocol violation. One from the sitagliptin group quitted for poor glycemic control. The baseline demographic and biochemical parameters of the subjects in the liraglutide group, metformin group and sitagliptin group were shown in Table 1. As expected because of randomization, the baseline characteristics were similar among the three groups in terms of age, diabetes duration, BMI, FBG, HbAlc, fast insulin, fast $\mathrm{C}$-peptide and plasma lipids.

\section{Glycemic control}

The mean values of HbA1c were significantly reduced in all the three groups over 24 -week treatment period $(\mathrm{P}<0.001)$. At the endpoint of 24 weeks, the mean HbA1c levels decreased from $8.8 \%$ to $7.1 \%$ with liraglutide, from $8.7 \%$ to $7.5 \%$ with metformin and from $8.6 \%$ to $7.4 \%$ with sitagliptin. The mean reduction of $\mathrm{HbAlc}$ values was greatest for the subjects treated with liraglutide $1.2 \mathrm{mg}$ daily (1.7\%) among all treatment groups at the endpoint, much greater than $1.2 \%$ with met- formin group $(\mathrm{P}=0.005)$, and $1.2 \%$ with sitagliptin group $(\mathrm{P}=$ $0.002)$. However, there was no significant difference between sitagliptin group and metformin group $(\mathrm{P}=0.999)$.

The mean values of FBG were significantly reduced in all the three groups over 24 -week treatment period $(\mathrm{P}<0.001)$. At the endpoint of 24 weeks, the mean reduction of $\mathrm{FBG}$ value from baseline was $2.2 \mathrm{mmol} / \mathrm{L}$ with liraglutide treatment, $1.7 \mathrm{mmol} / \mathrm{L}$ with metformin group and $1.6 \mathrm{mmol} / \mathrm{L}$ with sitagliptin. However, pairwise comparisons among the three treatment groups did not reach statistically significant difference $(\mathrm{P}>0.05)$.

Thirteen subjects of the 24 from liraglutide group achieved a target $\mathrm{HbA} 1 \mathrm{c}<7.0 \%$, compared with six of 25 from sitagliptin group $\left(\chi^{2}=4.694\right.$ and $\left.\mathrm{P}=0.042\right)$, and five of 26 from metformin group, respectively $\left(\chi^{2}=6.611\right.$ and $\left.\mathrm{P}=0.018\right)$. However, the percentage was similar between sitagliptin and metformin treated subjects. Among the patients who showed an HbA1c improvement from the liraglutide group, a clear linear relationship between $\mathrm{HbAlc}$ at baseline and the decrease in $\mathrm{HbA1c}$ was found (Spearman correlation $\mathrm{r}=0.810 ; \mathrm{P}<0.001$ ). Thus, higher baseline value of HbAlc predicts a greater decrease in HbAlc over 24 weeks with liraglutide. Whereas, those from the metformin group and sitagliptin group did not show this tendency. 
Table 2. Changes of Variables Related With Glycemic Control, Body Weight and Adipokines After 24-Week Treatment

\begin{tabular}{|c|c|c|c|c|c|}
\hline & Baseline & 24 weeks & $\begin{array}{l}\text { Mean change } \\
\text { from baseline }\end{array}$ & $\begin{array}{l}\text { P value (effect of } \\
\text { time) }\end{array}$ & $\begin{array}{l}\text { P value (effect of } \\
\text { treatment) }\end{array}$ \\
\hline \multicolumn{6}{|l|}{ HbAlc (\%) } \\
\hline Liraglutide & $8.8 \pm 1.0$ & $7.1 \pm 0.7$ & $1.7 \pm 0.5$ & $<0.001$ & LvsM 0.005 \\
\hline Metformin & $8.7 \pm 0.7$ & $7.5 \pm 0.6$ & $1.2 \pm 0.5$ & $<0.001$ & MvsS 0.999 \\
\hline Sitagliptin & $8.6 \pm 0.8$ & $7.4 \pm 0.6$ & $1.2 \pm 0.4$ & $<0.001$ & LvsS 0.002 \\
\hline \multicolumn{6}{|l|}{ FBG $(\mathrm{mmol} / \mathrm{L})$} \\
\hline Liraglutide & $9.3 \pm 1.6$ & $7.1 \pm 0.7$ & $2.2 \pm 1.1$ & $<0.001$ & LvsM 0.117 \\
\hline Metformin & $9.3 \pm 0.9$ & $7.7 \pm 0.8$ & $1.7 \pm 0.6$ & $<0.001$ & MvsS 0.969 \\
\hline Sitagliptin & $9.2 \pm 1.7$ & $7.6 \pm 1.2$ & $1.6 \pm 0.6$ & $<0.001$ & LvsS 0.068 \\
\hline \multicolumn{6}{|l|}{ Body weight (kg) } \\
\hline Liraglutide & $88.7 \pm 6.5$ & $83.9 \pm 6.0$ & $4.8 \pm 1.2$ & $<0.001$ & LvsM $<0.001$ \\
\hline Metformin & $88.2 \pm 7.6$ & $85.6 \pm 6.4$ & $2.6 \pm 1.4$ & $<0.001$ & MvsS 0.113 \\
\hline Sitagliptin & $88.9 \pm 6.2$ & $87.2 \pm 5.6$ & $1.8 \pm 1.4$ & $<0.001$ & LvsS $<0.001$ \\
\hline \multicolumn{6}{|l|}{ BMI $\left(\mathrm{kg} / \mathrm{m}^{2}\right)$} \\
\hline Liraglutide & $30.4 \pm 2.7$ & $28.8 \pm 2.7$ & $1.6 \pm 0.4$ & $<0.001$ & LvsM $<0.001$ \\
\hline Metformin & $30.2 \pm 2.4$ & $29.3 \pm 2.3$ & $0.9 \pm 0.4$ & $<0.001$ & MvsS 0.065 \\
\hline Sitagliptin & $30.6 \pm 3.2$ & $30.1 \pm 3.2$ & $0.6 \pm 0.4$ & $<0.001$ & LvsS $<0.001$ \\
\hline \multicolumn{6}{|c|}{ Waist circumstance $(\mathrm{cm})$} \\
\hline Liraglutide & $110.2 \pm 4.7$ & $103.2 \pm 4.5$ & $6.9 \pm 1.3$ & $<0.001$ & LvsM $<0.001$ \\
\hline Metformin & $109.7 \pm 4.2$ & $105.9 \pm 4.1$ & $3.8 \pm 2.0$ & $<0.001$ & MvsS 0.123 \\
\hline Sitagliptin & $110.1 \pm 3.8$ & $107.4 \pm 3.3$ & $2.7 \pm 1.9$ & $<0.001$ & LvsS $<0.001$ \\
\hline \multicolumn{6}{|c|}{ HOMA-IR (mol mIU/L²) } \\
\hline Liraglutide & $5.8 \pm 2.4$ & $3.1 \pm 1.0$ & $2.6 \pm 1.6$ & $<0.001$ & LvsM 0.054 \\
\hline Metformin & $5.3 \pm 2.1$ & $3.7 \pm 1.4$ & $1.7 \pm 1.0$ & $<0.001$ & MvsS 0.962 \\
\hline Sitagliptin & $5.8 \pm 2.4$ & $4.0 \pm 1.5$ & $1.8 \pm 1.1$ & $<0.001$ & LvsS 0.119 \\
\hline \multicolumn{6}{|c|}{ HOMA-B ( $\mu \mathrm{IU} / \mathrm{mmol})$} \\
\hline Liraglutide & $49.3 \pm 16.6$ & $56.7 \pm 17.7$ & $7.5 \pm 9.2$ & 0.001 & LvsM 0.998 \\
\hline Metformin & $45.2 \pm 18.4$ & $53.0 \pm 20.9$ & $7.9 \pm 9.8$ & $<0.001$ & MvsS 0.987 \\
\hline Sitagliptin & $51.3 \pm 18.8$ & $59.6 \pm 19.1$ & $8.3 \pm 9.7$ & $<0.001$ & LvsS 0.998 \\
\hline \multicolumn{6}{|l|}{ Leptin (ng/dL) } \\
\hline Liraglutide & $28.4 \pm 9.2$ & $26.5 \pm 7.7$ & $1.9 \pm 2.5$ & 0.001 & LvsM 0.892 \\
\hline Metformin & $29.7 \pm 5.6$ & $28.4 \pm 5.3$ & $1.2 \pm 4.9$ & 0.219 & MvsS 0.846 \\
\hline Sitagliptin & $27.6 \pm 7.9$ & $26.6 \pm 5.4$ & $1.0 \pm 5.4$ & 0.341 & LvsS 0.999 \\
\hline \multicolumn{6}{|c|}{ Adiponectin $(\mu \mathrm{g} / \mathrm{dL})$} \\
\hline Liraglutide & $6.5 \pm 2.2$ & $8.2 \pm 1.0$ & $1.7 \pm 1.5$ & $<0.001$ & LvsM 0.027 \\
\hline Metformin & $6.3 \pm 1.6$ & $6.5 \pm 1.2$ & $0.2 \pm 2.1$ & 0.565 & MvsS 0.980 \\
\hline Sitagliptin & $6.8 \pm 1.8$ & $7.2 \pm 1.0$ & $0.4 \pm 1.4$ & 0.156 & LvsS 0.016 \\
\hline \multicolumn{6}{|l|}{$\mathrm{L} / \mathrm{A}$ ratio $(\mathrm{ng} / \mu \mathrm{g})$} \\
\hline Liraglutide & $5.1 \pm 3.2$ & $3.3 \pm 1.2$ & $1.8 \pm 2.4$ & 0.001 & LvsM 0.159 \\
\hline Metformin & $5.1 \pm 1.8$ & $4.5 \pm 1.2$ & $0.6 \pm 2.0$ & 0.167 & MvsS 0.990 \\
\hline Sitagliptin & $4.5 \pm 2.1$ & $3.8 \pm 1.0$ & $0.7 \pm 1.9$ & 0.08 & LvsS 0.247 \\
\hline
\end{tabular}

Values are represented as mean \pm standard variation. $P$ value (effect of time) means comparison between endpoint of 24 weeks and baseline for each treatment. $P$ value (effect of treatment) means pairwise comparison of mean changes from baseline among the treatments. LvsM: liraglutide vs. metformin; MvsS: metformin vs. sitagliptin; LvsS: liraglutide vs. sitagliptin. 
Table 3. Overview of Adverse Events Over 24-Week Treatment

\begin{tabular}{llll}
\hline & Liraglutide & Metformin & Sitagliptin \\
\hline $\mathrm{n}$ & 24 & 26 & 25 \\
$\begin{array}{l}\text { Patients with one } \\
\text { or more AEs }\end{array}$ & $13(54.2)$ & $6(25.0)^{*}$ & $5(20.0)^{*}$ \\
$\begin{array}{l}\text { Hypoglycemia } \\
\text { Nausea }\end{array}$ & $2(8.3)$ & $1(3.8)$ & $1(4.0)$ \\
Vomiting & $8(33.3)$ & $2(7.7)^{*}$ & $1(4.0)^{*}$ \\
Diarrhea & $2(8.3)$ & $1(3.8)$ & $0(0)$ \\
Constipation & $2(12.5)$ & $1(3.8)$ & $1(4.0)$ \\
$\begin{array}{l}\text { Epigastric } \\
\text { abdominal pain }\end{array}$ & $3(12.5)$ & $2(7.7)$ & $1(4.0)$ \\
$\begin{array}{l}\text { Dizziness } \\
\begin{array}{l}\text { Influenza-like } \\
\text { symptoms }\end{array}\end{array}$ & $2(16.7)$ & $2(7.7)$ & $2(8.0)$ \\
Skin itching & $1(4.2)$ & $1(3.8)$ & $2(8.0)$ \\
\hline
\end{tabular}

Data are shown in $n(\%) .{ }^{*} \mathrm{P}<0.05$ for comparison with liraglutide group.

\section{BMI, weight, waist circumstance, waist hip ratio (WHR) and HOMA}

At the endpoint of 24 weeks, body weight significantly decreased in all the three treatment groups $(\mathrm{P}<0.001)$. Subjects treated with liraglutide lost on average $4.8 \pm 1.2 \mathrm{~kg}$ compared with a $2.6 \pm 1.4 \mathrm{~kg}$ loss in the metformin group $(\mathrm{P}<0.001)$ and $1.8 \pm 1.4 \mathrm{~kg}$ loss in the sitagliptin group $(\mathrm{P}<0.001)$; however, there was no statistically significant difference between the metformin and sitagliptin therapy groups $(\mathrm{P}=0.113)$. A total of $21.3 \%$ (16/75) subjects were high responders who lost $\geq 5 \%$ body weight in 24 weeks, $17.3 \%$ (13) of them in the liraglutide group compared with $2.7 \%$ (two) in the metformin group and, and only one subject in the sitagliptin group achieved this degree of weight loss.

At 24 weeks, BMI significantly decreased in all the three treatment groups $(\mathrm{P}<0.001$ for the effect of time). BMI decreased for $1.6 \pm 0.4 \mathrm{~kg} / \mathrm{m}^{2}$ from baseline in the liraglutide group compared with $0.9 \pm 0.4 \mathrm{~kg} / \mathrm{m}^{2}$ in metformin $(\mathrm{P}<0.001)$ and $0.6 \pm 0.4 \mathrm{~kg} / \mathrm{m}^{2}$ in the sitagliptin group $(\mathrm{P}<0.001)$. The analysis showed no statistically significant differences between the metformin and sitagliptin therapy arms $(\mathrm{P}=0.065)$. Comparable results were found for reduction in body waist circumference $(\mathrm{P}<0.001$ for the treatment/time effect). The maximum waist circumference reduction was noted in patients on liraglutide therapy followed by the metformin and sitagliptin therapy group patients (with treatment differences 6.9 $\pm 1.3,3.8 \pm 2.0$, and $2.7 \pm 1.9 \mathrm{~cm}$ respectively, $\mathrm{P}<0.001$ for the liraglutide vs. metformin, and $\mathrm{P}<0.001$ for the liraglutide vs. sitagliptin). All treatment interventions resulted in a nonsignificant reduction in WHR $(\mathrm{P}>0.05$ for the treatment/time effect).

In addition, significant HOMA-IR decrease and HOMA-B increase were observed in all the treatment groups $(\mathrm{P} \leq 0.001$ for effect of time). However, further analysis did not show statistical significance for pairwise comparisons $(\mathrm{P}>0.05$ for effect of treatment). Although other parameters showed an improvement after 24 weeks of therapy as systolic and diastolic blood pressure, total cholesterol, LDL cholesterol (LDL-c), HDL cholesterol (HDL-c) and TG, they did not reach statistical significance.

\section{Leptin, adiponectin, $\mathrm{L} / \mathrm{A}$ ratio and $\mathrm{hsCRP}$}

As shown in Table 2, the baseline leptin and adiponectin levels were similar among the three treatment groups. At the endpoint of 24 weeks, liraglutide administration resulted in significant reduction of leptin level from $28.4 \pm 9.2$ to $26.5 \pm 7.7$ $(\mathrm{P}=0.001)$, significant increase of adiponectin level from 6.5 \pm 2.2 to $8.2 \pm 1.0(\mathrm{P}<0.001)$ and significant reduction of $\mathrm{L} / \mathrm{A}$ ratio from $5.1 \pm 3.2$ to $3.3 \pm 1.2(\mathrm{P}=0.001)$. While the metformin and sitagliptin administration did not show this kind of significant changes in leptin, adiponectin and L/A ratio levels ( $P>0.05$ for the effect of time). After 24 weeks liraglutide administration, there was a significant increase in the adiponectin level $1.7 \pm 1.5 \mu \mathrm{g} / \mathrm{dL}$, much higher than metformin group $0.2 \pm$ $2.1 \mu \mathrm{g} / \mathrm{dL}(\mathrm{P}=0.027)$, and sitagliptin group $0.4 \pm 1.4 \mu \mathrm{g} / \mathrm{dL}(\mathrm{P}$ $=0.016)$. In addition, the changes of hsCRP levels of the three groups did not reach statistical significance $(\mathrm{P}>0.05$ for effect of time/treatment).

\section{Tolerability and safety}

In general, all the administrated drugs in the present study were well tolerated. The AEs of the three treatment groups were summarized in Table 3. The incidence of any treatmentrelated AEs was 54.2\% in patients with liraglutide, much higher than $23.1 \%$ with metformin $\left(\chi^{2}=5.120, \mathrm{P}=0.024\right)$ and $20.0 \%$ with sitagliptin $\left(\chi^{2}=6.151, \mathrm{P}=0.013\right)$. Gastrointestinal AEs were most commonly reported in the liraglutide group, especially nausea, occurring in $33.3 \%$ of patients in this group, while only $7.7 \%$ in metformin group $\left(\chi^{2}=5.128, \mathrm{P}=0.035\right)$ and only $4.0 \%$ in sitagliptin group $\left(\chi^{2}=7.027, \mathrm{P}=0.011\right)$. During the 24 weeks treatment period of the study, no patients experienced any major hypoglycemic episode. The incidence of hypoglycemia unconfirmed by glucose measurement was $8.3 \%, 3.8 \%$ and $4.0 \%$ for patients in liraglutide, sitagliptin and metformin groups, respectively $\left(\chi^{2}=0.630, \mathrm{P}=0.730\right)$. No significant difference was shown in the other AEs, including diarrhea, constipation, abdominal pain, dizziness, peripheral edema, influenza-like symptoms and skin itching. During the 24-week study, two subjects in liraglutide group and one in sitagliptin group withdrew from the study (reasons mentioned above).

\section{Discussion}

In this study, we explored the relationship of glycemic control, body weight, insulin resistance indexes (HOMA-IR and 
HOMA-B) and adipokines (leptin and adiponectin) in newly diagnosed overweight or obese T2DM patients at the beginning and 24 weeks after the initiation of liraglutide, sitagliptin or metformin treatment in daily clinical practice.

Administration of liraglutide, metformin or sitagliptin all resulted in significant improvements in glycemic control. It was also demonstrated that liraglutide was superior to metformin and sitagliptin in terms of achieving target $\mathrm{HbA} 1 \mathrm{c}$ values and sustaining weight loss after 24-week treatment, but not to the incidence of AEs. Although there were minor differences, there was no significant difference between metformin and sitagliptin administration with regard to glycemic control, weight loss and AEs. Moreover, liraglutide administration showed beneficial effects in reducing leptin levels, L/A ratio and elevating adiponectin levels compared to the metformin and sitagliptin administration.

GLP-1 is an incretin hormone physiologically secreted by intestinal L cells, mainly in response to food intake. Its effects include glucose-dependent stimulation of insulin secretion and glucagon reduction, slowing gastrointestinal motility and increasing satiety leading to reduced food intake [7]. In animal models, there is evidence that it also promotes $\beta$-cell proliferation and probably neogenesis, while reducing apoptosis [12, 13]. Our study supports previous data on liraglutide improvements in glucose control and weight reduction. HbA1c improvement was directly related to basal $\mathrm{HbA} 1 \mathrm{c}$ in concordance with previous studies [9-11]. As indicated in our study, greater proportion of patients treated with liraglutide reached the target level of $\mathrm{HbA} 1 \mathrm{c}<7 \%$ than the other two agents, without accompanying an increased risk of hypoglycemia.

HOMA is a widely validated clinical and epidemiological tool for the estimation of insulin resistance (HOMA- IR) and $\beta$-cell function (HOMA-B). The effect of GLP-1 administration on HOMA has been previously studied $[14,15]$. In our study, all the three kinds of treatment showed statistically significantly lower insulin resistance and higher insulin secretion compared with baseline. These results agree with previous clinical studies with GLP-1 analogs. Improvement in glucotoxicity and lipotoxicity under liraglutide treatment could explain HOMA amelioration. However, incretin-based treatment has not been shown to have superior effect on HOMA-IR and HOMA-B parameters over metformin. Extensive clinical trials are required to evaluate $\beta$ cell recovery in T2DM under GLP-1 treatment.

It is well recognized that abdominal obesity plays a central role in the development of insulin resistance and contributes significantly to the progression of T2DM. Recently, attention has focused on the role of visceral adipose tissue due to the synthesis and release of a number of adipokines from adipocytes [3-5]. In T2DM, increased visceral adipose tissue disturbs adipokines secretion and leads to a low-grade chronic inflammatory state mediated by the infiltration of macrophages into adipose tissue. This inflammatory state is found to be associated with insulin resistance. Visceral adipose tissue functions as a paracrine and an endocrine organ, secreting a number of adipokines, such as leptin and adiponectin. These molecules (leptin and adiponectin) have been linked to the pathogenesis of the metabolic syndrome and cardiovascular disease. In diabetic patients, serum adiponectin levels are decreased, while leptin levels are elevated $[16,17]$. This imbalance in the inflammatory state leads to dysfunction of the endothelial cells, promoting the loss of their vasodilatory, antithrombotic, and antiatherogenic properties, apparently a widespread biological response in humans.

Studies evaluating liraglutide effects on adipokines and biomarkers of cardiovascular risk were rarely reported in daily clinical practice. Only partial results have been published in clinical trials $[15,18]$. To our knowledge, no studies have addressed the effect of liraglutide on adipokines in newly diagnosed T2DM patients. Our study demonstrated the beneficial effects of liraglutide administration in reducing leptin and elevating adiponectin levels. No significant changes were observed after metformin and sitagliptin administration. Leptin has been recently described as an important modulator of the response to dynamic meal related signals with serum GLP-1 effects [19]. However, the detail mechanisms of liraglutide involved in adipokines regulation remain to be elucidated by more studies.

In conclusion, liraglutide treatment during 24 weeks in newly diagnosed T2DM patients led to reduction of BMI and improvement of glucose control and insulin sensitivity and resistance parameters. Additionally, circulating levels of adipokines could play an important role in GLP-1 treatment.

\section{Conflict of Interest}

None.

\section{References}

1. Quan W, Jo EK, Lee MS. Role of pancreatic beta-cell death and inflammation in diabetes. Diabetes Obes Metab. 2013;15(Suppl 3):141-151.

2. Matsuda M, Shimomura I. Roles of oxidative stress, adiponectin, and nuclear hormone receptors in obesity-associated insulin resistance and cardiovascular risk. Horm Mol Biol Clin Investig. 2014;19(2):75-88.

3. Richardson VR, Smith KA, Carter AM. Adipose tissue inflammation: feeding the development of type 2 diabetes mellitus. Immunobiology. 2013;218(12):1497-1504.

4. Mraz M, Haluzik M. The role of adipose tissue immune cells in obesity and low-grade inflammation. J Endocrinol. 2014;222(3):R113-127.

5. Cao H. Adipocytokines in obesity and metabolic disease. J Endocrinol. 2014;220(2):T47-59.

6. Inzucchi SE, Bergenstal RM, Buse JB, Diamant M, Ferrannini E, Nauck M, Peters AL, et al. Management of hyperglycemia in type 2 diabetes: a patient-centered approach: position statement of the American Diabetes Association (ADA) and the European Association for the Study of Diabetes (EASD). Diabetes Care. 2012;35(6):1364-1379.

7. Tomkin GH. Treatment of type 2 diabetes, lifestyle, GLP1 agonists and DPP4 inhibitors. World J Diabetes. 2014;5(5):636-650.

8. Buturovic BA, Ristic LB, Narancic AM. Effects of Teraphy with Basal Insulin Analogues Combined with GLP 
1 Analogues and Metformin in the Treatment of Obese Patients with Poorly Regulated Postprandial Glycemia. Med Arch. 2014;68(5):313-316.

9. Ahmann A, Rodbard HW, Rosenstock J, Lahtela JT, de Loredo L, Tornoe K, Boopalan A, et al. Efficacy and safety of liraglutide versus placebo added to basal insulin analogues (with or without metformin) in patients with type 2 diabetes: a randomized, placebo-controlled trial. Diabetes Obes Metab. 2015.

10. Li CJ, Yu Q, Yu P, Zhang QM, Ding M, Liu XJ, Yu DM. Efficacy and safety comparison of add-on therapy with liraglutide, saxagliptin and vildagliptin, all in combination with current conventional oral hypoglycemic agents therapy in poorly controlled Chinese type 2 diabetes. Exp Clin Endocrinol Diabetes. 2014;122(8):469-476.

11. Charbonnel B, Steinberg H, Eymard E, Xu L, Thakkar P, Prabhu V, Davies MJ, et al. Efficacy and safety over 26 weeks of an oral treatment strategy including sitagliptin compared with an injectable treatment strategy with liraglutide in patients with type 2 diabetes mellitus inadequately controlled on metformin: a randomised clinical trial. Diabetologia. 2013;56(7):1503-1511.

12. Miao XY, Gu ZY, Liu P, Hu Y, Li L, Gong YP, Shu H, et al. The human glucagon-like peptide-1 analogue liraglutide regulates pancreatic beta-cell proliferation and apoptosis via an AMPK/mTOR/P70S6K signaling pathway. Peptides. 2013;39:71-79.

13. Shimoda M, Kanda Y, Hamamoto S, Tawaramoto K, Hashiramoto M, Matsuki M, Kaku K. The human glucagonlike peptide-1 analogue liraglutide preserves pancreatic beta cells via regulation of cell kinetics and suppression of oxidative and endoplasmic reticulum stress in a mouse model of diabetes. Diabetologia. 2011;54(5):1098-1108.

14. Feng P, Yu DM, Chen LM, Chang BC, Ji QD, Li SY, Zhu $M$, et al. Liraglutide reduces the body weight and waist circumference in Chinese overweight and obese type 2 diabetic patients. Acta Pharmacol Sin. 2015;36(2):200208.

15. Diaz-Soto G, de Luis DA, Conde-Vicente R, Izaola-Jauregui $\mathrm{O}$, Ramos $\mathrm{C}$, Romero E. Beneficial effects of liraglutide on adipocytokines, insulin sensitivity parameters and cardiovascular risk biomarkers in patients with Type 2 diabetes: a prospective study. Diabetes Res Clin Pract. 2014;104(1):92-96.

16. Zaletel J, Barlovic DP, Prezelj J. Adiponectin-leptin ratio: a useful estimate of insulin resistance in patients with Type 2 diabetes. J Endocrinol Invest. 2010;33(8):514-518.

17. Coimbra S, Brandao Proenca J, Santos-Silva A, Neuparth MJ. Adiponectin, leptin, and chemerin in elderly patients with type 2 diabetes mellitus: a close linkage with obesity and length of the disease. Biomed Res Int. 2014;2014:701915.

18. Suzuki D, Toyoda M, Kimura M, Miyauchi M, Yamamoto $\mathrm{N}$, Sato H, Tanaka E, et al. Effects of liraglutide, a human glucagon-like peptide-1 analogue, on body weight, body fat area and body fat-related markers in patients with type 2 diabetes mellitus. Intern Med. 2013;52(10):1029-1034.

19. Williams DL, Baskin DG, Schwartz MW. Leptin regulation of the anorexic response to glucagon-like peptide-1 receptor stimulation. Diabetes. 2006;55(12):3387-3393. 